\title{
Treatments for Age-related Macular Degeneration
}

\author{
Winfried MK Amoaku \\ Associate Professor/Reader and Honorary Consultant Ophthalmologist, Division of Ophthalmology and Visual Sciences, \\ University Hospital, Queen's Medical Centre, Nottingham
}

\begin{abstract}
Age-related macular degeneration (AMD) is the most common cause of irreversible visual loss in the developed world. There are two types of AMD: dry and wet. The exact cause of the disease is unknown, but is thought to result from interplay of genetic and environmental factors. Smoking, hypertension and low intake and/or low levels of serum antioxidants are known risk factors for both forms. As yet there are no treatments available for the dry form of AMD. Smoking cessation and healthy lifestyles are important in the prevention of progression of AMD. Progression of early stages of AMD to advanced forms is slowed by regular intake of ocular nutritional supplements as found in the Age-Related Eye Disease Study (AREDS) Study. Recently, there have been significant breakthroughs in the treatment of wet or neovascular AMD, including photodynamic therapy (PDT), intravitreal injections of anti-vascular endothelial growth factor (anti-VEGF) drugs, including pegaptanib, ranibizumab and bevacizumab. Newer agents including aflibercept and anti-platelet-derived growth factor (anti-PDGF) compounds are under investigation. The current gold standard for treatment of wet AMD is intravitreal injections of ranibizumab. Combination therapy with PDT and anti-VEGF agents is useful especially in idiopathic polypoidal choroidopathy (IPCV) or when anti-VEGF monotherapy has failed. Rapid referral of patients with neovascular AMD is important as the visual outcome is dependent on the visual acuity at treatment. Diagnostic confirmation with retinal imaging, particularly fundus fluorescein angiography and optical coherent tomography, are required before treatment commencement. The new treatments for wet AMD are repetitive, and have significant service implications. These implications occur irrespective of which antiVEGF agent is adopted. Visual rehabilitation is still important in the management of AMD.
\end{abstract}

\section{Keywords}

Macular degeneration, age-related, ocular nutritional supplements, laser photocoagulation, verteporfin, photodynamic therapy (PDT), pegaptanib, ranibizumab, bevacizumab, vascular endothelial growth factor (VEGF), VEGF Trap

Disclosure: Winfried MK Amoaku has received travel grants and speaker's honoraria from Allergan, Novartis Pharma and Pfizer, served on advisory boards for Allergan, Novartis and Pfizer and participated in research sponsored by Bausch and Lomb, Novartis and Pfizer for which his institution received funding from these organisations. Acknowledgements: The author has had access to unpublished study data from Pfizer and Novartis. The views expressed in this article may reflect, but not necessarily represent, those of any of these organisations.

Received: 16 May 2010 Accepted: 22 June 2010 Citation: European Ophthalmic Review, 2010;4:92-100 DOI: 10.17925/EOR.2010.04.01.92

Correspondence: Winfried MK Amoaku, Associate Professor/Reader and Honorary Consultant Ophthalmologist, Division of Ophthalmology and Visual Sciences, University Hospital, Queen's Medical Centre, Nottingham, NG7 2UH, UK. E: winfried.amoaku@nottingham.ac.uk

Age-related macular degeneration (AMD) is the most common cause of irreversible visual loss in the western world. ${ }^{1-5}$ Broadly, there are two types of AMD: dry or non-exudative AMD, and wet or exudative AMD. The dry form is characterised by drusen formation and a slow, progressive focal atrophy of the macular retinal pigment epithelium (RPE) and inner choroid. This in turn leads to secondary atrophy of the overlying photoreceptors. Over time, this focal atrophy spreads to involve large parts of the macula, leading to so-called geographic atrophy. The initial symptoms reported by patients include gaps in images or words and slow progressive blurring. With time, these focal defects enlarge to produce a central scotoma.

Wet AMD is characterised by the growth of abnormal new vessels arising from the inner choroid. These new vessels, called choroidal new vessels (CNVS), penetrate Bruch's membrane and grow under the RPE or, with further penetration of the RPE, to the potential space under the neuroretina (photoreceptors). ${ }^{5-9}$ The clinical features include retinal and subretinal haemorrhages, retinal pigment epithelial detachment (serous or haemorrhagic), exudates and fibrous proliferation in the late stage. The majority of CNVs in AMD involve the subfoveal area ${ }^{10}$ and are stimulated by growth factors such as vascular endothelial growth factor (VEGF) and fibroblastic growth factor (FGF). Other growth factors have also been demonstrated to be associated with excised CNVs. ${ }^{11-17}$ These new vessels leak fluid, lipid and blood, causing elevation of the retina. Patients often complain of distortion early on in the course of the disease before rapid visual loss occurs. Unless treated early, irreversible damage to photoreceptors occurs quickly (often within a few weeks). ${ }^{10}$

Dry AMD accounts for $85-95 \%$ of all AMD, but is responsible for $10-20 \%$ of severe visual loss in AMD, while the wet type occurs in $10-15 \%$ of all AMD but accounts for $90 \%$ of the visual loss in AMD..$^{1,2,4}$

In the Beaver Dam Eye Study in the US, the prevalence of advanced AMD increased from $0.1 \%$ at $43-54$ years of age to $7.1 \%$ among those 75 years of age or older. ${ }^{2}$ In a similar study conducted in Australia, $0 \%$ 
of people under 55 years of age had advanced $\mathrm{AMD}_{4}^{4}$ however, this increased to $18.5 \%$ over 85 years of age. It is estimated that there are approximately 215,000 people with wet AMD in the UK, ${ }^{18}$ with an estimated incidence of wet AMD in the UK of $26,000 .{ }^{19}$ It is further accepted that approximately $10 \%$ of patients with wet AMD in the first eye will develop similar pathology in the other eye with every passing year. ${ }^{20}$ With current demographic changes with a skew towards a more elderly population, the number of people with $\mathrm{AMD}$ is likely to increase even further, with marked health economic implications. ${ }^{5}$

\section{Risk Factors}

The exact cause of AMD remains elusive. AMD is probably a multifactorial disease affected by both genetic and environmental factors. This may account for the wide variation in phenotypes.

A number of large epidemiological studies have identified a number of risk factors. Increasing age is an undoubted risk factor; however, this, unfortunately, is not modifiable. ${ }^{21}$ Smoking has been shown to increase the risk of AMD by between three- and four-fold. ${ }^{22,23}$ Therefore, smoking cessation regimens offer the prospect of dramatically reducing the incidence of severe visual loss secondary to AMD. The finding of macular hard drusen (small yellow sub-retinal deposits) increases dramatically with advancing age without significant risk of advanced AMD; however, the possession of the large, soft drusen increases the risk of advanced AMD by between five- and 10 -fold. ${ }^{20,24}$ In addition, the finding of advanced AMD (e.g. CNV secondary to wet AMD, or geographic atrophy) in one eye means that there is a $50 \%$ risk of the fellow eye developing a similar lesion over five years. ${ }^{20,24}$ Other factors such as obesity, diets high in saturated fat content and high alcohol consumption, all of which result in low body antioxidant levels, may predispose to the development and progression of AMD.

In 2000, Mullins et al. demonstrated that drusen in AMD had a significant inflammatory element. ${ }^{25}$ This was linked to the major breakthrough in our understanding of the genetic risks of AMD pathogenesis in 2005. ${ }^{26}$ The discovery that the complement system in some way played a role in AMD confirmed the existence of an immune-related mechanism in the pathogenesis of the disease. Several genes are now known to play an important role in the pathogenesis of $\mathrm{AMD}$ : the complement factor $\mathrm{H}(\mathrm{CFH})$ gene on chromosome $1,{ }^{26-29}$ a region on chromosome 10 harbouring the gene for protein LOC387715, the serine protease HTRA1 gene, ${ }^{30-32}$ the complement factor B (CFB) and complement component 2 (CC2) genes on chromosome $6^{33}$ and the complement component 3 (CC3) gene on chromosome 19. Treatments based on genetic modifications in humans are yet to be tested.

\section{Diagnosis}

Diagnosis of AMD is dependent on clinical features and ancillary investigations. In particular, retinal imaging with fundus fluorescein angiography (FFA) and optical coherence tomography (OCT) are an integral part of patient management and are required for confirmation of diagnosis and for monitoring response to therapy, especially in wet AMD. ${ }^{34,35}$ As such, treatments should not be initiated in any patient without adequate imaging with FFA and OCT. Indocyanine green angiography (ICG-A) provides information that complements that derived from FFA to optimise the management of AMD patients. ${ }^{35}$ Angiographic subtypes of wet AMD include classic, occult or mixed lesions. Retinal angiomatous proliferations (RAPS) represent anastomoses between the retinal and choroidal vasculature and occur secondary to CNVs, especially of the occult type. ${ }^{36-39}$ Irrespective of whether they arise from the choroidal or retinal vasculature, RAPs leak and alter the behaviour of the CNV Iesion to therapy..$^{40}$ Idiopathic polypoidal choroidopathy (IPCV) is an atypical form of neovascular AMD in which highly exudative lesions with haemorrhagic pigment epithelial detachments are seen adjacent to the optic disc. These lesions are best visualised with ICG-A, which shows dilated grapelike or polypoidal complexes in the inner choroid. ${ }^{41}$

\section{Treatments for Dry Age-related Macular Degeneration}

No treatment exists for dry AMD to date. However, there has recently been growing interest in developing treatments for dry AMD following significant improvements in therapies for wet AMD.

At present, progression of the disease may be modified with ocular nutritional supplements. ${ }^{42,43}$ Subgroup analysis of a large multicentre, randomised, placebo-controlled trial (the Age-Related Eye Disease Study [AREDS]) suggested that progression from early to advanced AMD could be reduced by as much as $25 \%$ in high-risk patients by daily supplements of vitamins C (500mg) and E (400IU), zinc (80mg) and beta carotene (15mg). ${ }^{43}$ On the basis of these results, the AREDS research group recommend that people over 55 years of age should undergo dilated fundus examinations to determine their risk of developing advanced AMD. Those with high-risk characteristics should consider taking supplements and antioxidants plus zinc. Importantly, however, this formulation is not recommended for smokers because beta carotene has been shown to increase the risk of lung cancer. There is also evidence to suggest that those individuals with a high intake of the macular pigments lutein and zeaxanthin were at reduced risk of AMD. There is an ongoing large international trial investigating the value of lutein/zeaxanthin and omega-3 long-chain polyunsaturated fatty acid supplementation in AMD (AREDS 2 trial); this trial is not expected to report for a number of years. ${ }^{44}$

\section{Treatments for Wet Age-related Macular Degeneration}

Until recently, the main modalities for treatment of wet AMD were physical and included focal laser photocoagulation in small extrafoveal CNVs and photodynamic therapy (PDT) for subfoveal/juxtafoveal lesions. Pharmacological treatments have evolved for wet AMD based on our recently improved understanding of the pathophysiology of the disease and similarities to vascular biology in cancer therapy.

\section{Laser Photocoagulation}

The Macular Photocoagulation Study Group (MPSG) for wet AMD has shown that laser photocoagulation with conventional thermal laser is effective for extrafoveal lesions, and destroys the CNV before ingrowth to the fovea has occurred..$^{45}$ The burns must be confluent, totally covering the lesion, extending into the normal retina surrounding the lesion and of adequate intensity to fully ablate the CNV without rupturing Bruch's membrane. However, the effectiveness of this treatment is limited by the resultant scotoma in the visual field, by the high recurrence rate of CNV and by the fact that only small classic CNV that are extrafoveal at presentation can be treated in this way. ${ }^{45,46}$ Practically, only a few patients present with these small classic extrafoveal lesions; however, it is still the treatment of choice for this small group of patients. 
Laser photocoagulation is not recommended for eyes with subfoveal CNV because of the immediate visual loss that results from foveal photoreceptor and RPE damage. Laser photocoagulation is also less useful and not generally recommended for juxtafoveal CNV. This is because of the potential immediate collateral damage to the fovea from the laser burn, or later encroachment of the scar on the fovea. ${ }^{47,48}$

\section{Photodynamic Therapy with Verteporfin}

PDT with verteporfin destroys CNV without damaging the overlying neurosensory retina, thereby allowing subfoveal lesions to be treated. ${ }^{49}$ Verteporfin PDT acts through occlusion of the newly formed, actively growing vessels of the CNV lesion while avoiding damage to the other choroidal blood vessels and the RPE (within limits). In clinical trials, PDT with verteporfin has been shown to be effective in reducing visual loss compared with placebo-treated patients with classic and predominantly classic subfoveal CNV secondary to AMD. ${ }^{49,50}$ In addition, the Verteporfin in Photodynamic therapy (VIP) study showed that after two years, PDT with verteporfin significantly reduced the risk of moderate to severe visual loss in patients with occult and no classic CNV. ${ }^{51}$ Similar findings have been reported for CNV secondary to myopia. ${ }^{52}$ However, the EU licence for the use of PDT in the treatment of occult CNV was subsequently revoked: the Committee for Medicinal Products for Human Use (CHMP) recommended deletion of the indication of visudyne PDT in patients with occult CNV as the risk-benefit profile was no longer considered favourable following reports from the Visudyne PDT in Occult CNV (VIO) study (see EU Marketing Authorisation Number $\mathrm{EU} / 1 / 00 / 140 / 001)$.

\section{Anti-vascular Endothelial Growth Factors}

Although several growth factors, including FGF, platelet-derived growth factor (PDGF) and placental growth factor (PIGF) have been identified in CNV, VEGF is thought to be the most significant growth factor in the development and growth of CNV in wet AMD. ${ }^{11-17}$ This has led to the development of molecular inhibitors of VEGF, including pegaptanib (Macugen), ranibizumab (Lucentis) and others, as treatments for wet AMD.

\section{Pegaptanib Sodium}

Pegaptanib (Macugen, Pfizer/OSI Eyetech) is a pegylated modified oligonucleotide, an aptamer, with a molecular weight of 20kD that binds isoform 165 of VEGF (VEGF 165), inhibiting its activity. VEGF 165 is the isoform of VEGF that was originally thought to be preferentially increased in pathological neovascularisations $\mathrm{s}^{53}$ and CNV.

\section{Clinical Trials}

The VEGF Inhibition Study in Ocular Neovascularisation (VISION) trial ${ }^{54,55}$ was a multicentre, prospective, randomised, dose-ranging, double-blind, controlled trial of pegaptanib at doses of 0.3, 1.0 and $3.0 \mathrm{mg}$ or sham injections administered every six weeks. The study was run in two concurrent arms (US Food and Drug Administration [FDA] regulations) over an initial period of 48 weeks, subsequently extended to two years. The results showed that more patients who received pegaptanib $0.3 \mathrm{mg}$ compared with sham injections maintained their vision and, furthermore, that severe visual loss was reduced. Vision improved by 15 letters in 6\% of patients and was maintained in 33\%. Pegaptanib (Macugen) at $0.3 \mathrm{mg}$ is therefore effective in the treatment of all subtypes of CNV secondary to $\mathrm{AMD}$.

\section{Use of Pegaptanib in Routine Clinical Practice}

Pegaptanib (Macugen) was licensed for use in the US in December 2004. It was licensed by the European Medicines Agency (EMEA) at a dose of 0.3mg in February 2006, and launched in the UK in May 2006. Substantial experience of its use was accrued from clinical trials and private medical practice in the EU and worldwide prior to approvals and subsequently. ${ }^{56-58}$

The VISION entry criteria may provide a guide for clinical practice. ${ }^{54}$ In that trial pegaptanib was used to treat lesions of any subtype with the greatest linear diameter of 12 disc diameters or fewer and visual acuities (VAS) between approximately 6/12 and 1/60. CNV lesions of the minimally classic and occult type were required to demonstrate progression (of different indicators) before treatment. Pegaptanib is administered at six-weekly intervals.

\section{Safety in Clinical Practice}

The two-year safety data from VISION are reassuring. Injection-related endophthalmitis $(0.16 \%$ /injection) in the first year was attributed to violations in the injection preparation protocol. ${ }^{55}$ Significant reductions in endophthalmitis rates were achieved when these violations were curtailed.

There have been some reports of severe systemic allergic reactions associated with intravitreal pegaptanib injections, which may occur up to one hour following the injection. ${ }^{59}$ Similarly, there have been a few reports of retinal pigment epithelial rips following treatment with pegaptanib. However, as these can occur spontaneously or following laser treatment, their occurrence cannot be attributed to the administration of pegaptanib. ${ }^{60}$

The use of pegaptanib has gone into decline recently following the availability of ranibizumab and bevacizumab.

\section{Ranibizumab}

\section{Clinical Efficacy and Clinical Trials}

Randomised, double-blind, controlled, multicentre phase III trials (ANCHOR, MARINA and PIER) have evaluated the efficacy of intravitreal ranibizumab in the treatment of neovascular AMD. ${ }^{61-64}$ Other trials include SUSTAIN, SAILOR and EXCITE.

The MARINA study compared ranibizumab $0.3 \mathrm{mg} / \mathrm{month}(\mathrm{n}=238)$ or $0.5 \mathrm{mg} / \mathrm{month}(\mathrm{n}=240)$ with sham injections $(n=238)$ in eyes with minimally classic or occult subfoveal neovascular AMD for two years. ${ }^{61}$ The ANCHOR study compared ranibizumab $0.3 \mathrm{mg} / \mathrm{month}$ $(n=140)$ or $0.5 \mathrm{mg} /$ month $(n=140)$ with verteporfin PDT at baseline and repeated every three months as required $(n=143)$ over two years in patients with predominantly classic CNV. .2,63 $^{2}$

The study outcomes were similar in the ANCHOR and MARINA trials: VA was at least maintained in $94-96 \%$ of eyes receiving ranibizumab at one year and in 90-92\% at two years compared with 62 and 53\%, respectively, at one and two years in PDT-treated controls $(p<0.001) .61,64$ VA improved by 15 or more letters in $25-40 \%$ of patients receiving ranibizumab at one year and $26-33 \%$ at two years in these trials compared with 5-6 and $4 \%$ at one and two years, respectively, in the controls $(p<0.001) .62,65$ An overall VA gain of seven to 11 letters at one year and five to seven letters at two years was achieved compared with VA loss of 10 and 15 letters at one and two years, respectively, in the sham injection group and a loss of 10 and 9.8 
letters at one and two years, respectively, in the PDT group. ${ }^{61-65}$ Severe VA loss ( $>30$ letters) occurred in $<1 \%$ of the ranibizumab-treated patients compared with $13 \%$ of controls at one year and in 3 and $23 \%$, respectively, at two years in the MARINA study $(p<0.001)$, ${ }^{61,65}$ while in the ANCHOR Study severe visual loss (>30 letters) occurred in only $1.4 \%$ of the patients treated with the $0.3 \mathrm{mg}$ dose and none in the $0.5 \mathrm{mg}$ group compared with $16.1 \%$ in the PDT group. ${ }^{62,64}$

Subgroup analyses indicated that the VA changes were consistent across all sexes, ages, lesion types and baseline lesion sizes. ${ }^{66,67}$ The most important predictors of VA outcomes were baseline VA score, CNV lesion size and patient age.$^{66,67}$ Angiographic and OCT analyses of the MARINA study have shown consistent improvement in FFA leakage and OCT thickness in eyes treated with ranibizumab compared with sham-treated eyes. ${ }^{68}$

Quality of life (QOL) analyses in the MARINA study showed that ranibizumab-treated patients had significantly improved QoL after treatment compared with the sham-treated group. ${ }^{69}$ Similarly, QoL was significantly better at up to 24 months in the ranibizumab-treated group compared with the PDT group..$^{70}$ This was especially so for near activities, distance activities and dependency $(p<0.001){ }^{69,70}$

The PIER study was designed to determine whether a less frequent dosing regime after initiation with ranibizumab every month for three doses was enough to prevent visual loss in patients with subfoveal wet AMD. ${ }^{63}$ It compared ranibizumab $0.3 \mathrm{mg} / \mathrm{month}(\mathrm{n}=60), 0.5 \mathrm{mg} / \mathrm{month}$ $(n=61)$ and sham injections $(n=63)$ given for three doses, and identical single doses repeated at three-monthly (quarterly) intervals for two years in all types of CNV. The results from this study indicated that the quarterly dosage of ranibizumab after the initial loading was not enough to maintain the VA gain attained after month three compared with continued monthly dosing. However, the VA outcome was still significantly better in the ranibizumab-treated group compared with the 16-letter VA loss in the sham-treated group. ${ }^{63}$

The SUSTAIN study was an open-label, multicentre phase IIIb/IV trial where ranibizumab at 0.3 or $0.5 \mathrm{mg} / \mathrm{month}$ was administered for three doses followed by dosing as required based on VA and OCT criteria at monthly intervals for 12 months. The interim analysis (which included 71 patients) showed that the average number of treatments required in the 12 months was 5.3. ${ }^{71}$ Similarly, the final report showed that an average total of 5.6 injections were required over 12 months (Novartis, data on file). However, the VA gain achieved with the SUSTAIN regime was not as impressive as that achieved with regular monthly dosing as in the ANCHOR and MARINA studies. ${ }^{11}$ Subgroup analysis of the SUSTAIN data showed that there were three distinct responder groups: the first group maintained their initial VA gain with no or only minimal re-treatment, the second required more injections to maintain the initial gain, which otherwise deteriorated, and the third group had progressive VA loss in the maintenance treatment phase (Novartis, data on file).

Results from SAILOR, a 12-month phase IIIb study of monthly administration as required of ranibizumab after an initial three monthly doses according to protocol-defined VA or OCT changes, showed that there was reduced benefit at 12 months compared with the monthly dosing regimes. However, as baseline VA decreased, the degree of VA improvement was better. ${ }^{72,73}$ The results from EXCITE, in which the three initial monthly loading doses of ranibizumab (0.3 or
$0.5 \mathrm{mg}$ ) followed by monthly or quarterly doses of 0.3mg ranibizumab, show that in the quarterly treated group the VA improved by 4.0 letters in the $0.3 \mathrm{mg}$ group and 2.8 in the $0.5 \mathrm{mg}$ group. Subgroup analysis identified responder groups similar to those in the SUSTAIN study (Novartis, data on file).

When data from the four studies (MARINA, ANCHOR, PIER and SAILOR) were combined, the acute thromboembolic event (ATE) rate ${ }^{74}$ was $2.7 \%$, which was not statistically different from that in the control group. ${ }^{75,76}$ In the second year of the MARINA study, the rate of ATE events was similar in patients treated with ranibizumab $0.5 \mathrm{mg}(2.6 \%)$ and patients in the control arm (3.2\%). ${ }^{65}$ Analyses of pooled two-year data from the MARINA, ANCHOR and PIER trials showed that there were no safety concerns as the rate of ATE was $4.1 \%$ after year two, which was comparable to that in the control group. There was a nonstastistically significant difference towards a higher rate of stroke in the $0.5 \mathrm{mg}$ ranibizumab group compared with controls; no such difference was noted for myocardial infarctions (MIS). ${ }^{77}$ In further meta-analysis of the MARINA, ANCHOR, FOCUS, PIER and SAILOR data there was no statistically significant difference in ATES, although prior stroke was the significant risk factor for stroke in cohort 1 of the SAILOR study.

Bilateral intravitreal administration of ranibizumab has not been formally investigated in trials. Theoretically, bilateral simultaneous administration of ranibizumab could lead to an increased systemic exposure, with a possible increase in the risk of systemic adverse events. So far, post-marketing clinical experience of bilateral simultaneous administration of intravitreal ranibizumab has not highlighted any safety concerns as long as asepsis is observed and instruments are changed between the two eyes.

\section{Contraindications}

Hypersensitivity to ranibizumab or to any of the excipients in Lucentis is a contraindication. Although not investigated, it is possible that a hypersensitivity reaction to ranibizumab will result in a similar reaction to bevacizumab. Similarly, ranibizumab should be avoided in eyes with active severe intraocular inflammations as such administration may aggravate the inflammation. It is also advised that ranibizumab should not be administered concurrently with other anti-VEGF agents (systemic or ocular) as this will result in increased systemic exposure and potential toxicity. Ranibizumab is also to be avoided in eyes with rhegmatogenous retinal detachment and grade 4 macular holes. ${ }^{78}$

\section{Dosage and Administration/Different Regimes}

In the US, the recommended dosing regime for ranibizumab is $0.5 \mathrm{mg}$ delivered by intravitreal injection monthly. After the first four doses, treatment may be given quarterly or as required. ${ }^{38}$

In the EU, including the UK, the recommended dose of ranibizumab in wet AMD is $0.5 \mathrm{mg}$ given intravitreally. The recommended regime is initiation with three monthly doses, after which, in the maintenance phase, treatment is given as required based on visual acuity outcomes. ${ }^{78}$ In the UK in particular, the Royal College of Ophthalmologists (RCOphth) guide advises treatment as in the summary of product characteristics, with treatment guided by LogMAR visual acuity and OCT changes. ${ }^{79}$

\section{Current Status}

Ranibizumab (Lucentis) has been licensed by the EMEA and FDA for the treatment of neovascular membrane of all lesion types. In the UK, 
the National Institute for Health and Clinical Excellence (NICE), after a protracted evaluation, has recommended ranibizumab in the treatment of eyes with subfoveal CNV of all lesion types secondary to $A M D .{ }^{80}$ The VA restrictions in the guidance were based on the inclusion criteria of the pivotal ranibizumab studies. However, the RCOphth guide advises clinicians to offer treatment in all eyes with VA better than LogMAR 1.2, i.e there are no upper VA limitations. ${ }^{79}$ RAPS that occur as part of CNV respond better to ranibizumab than to PDT or focal laser photocoagulation, although there are no randomised

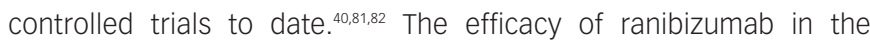
treatment of IPCV is yet to be fully established; however, The Asia SUMMIT (EVEREST) study suggests that combination therapy with PDT and ranibizumab is more efficacious than monotherapy in the treatment of IPCV (see below) (Novartis, data on file).

Emerging evidence suggests that ranibizumab is also effective in the treatment of subfoveal CNV secondary to causes other than AMD, e.g. myopia, angioid streaks, idiopathic and inflammation. ${ }^{83-85}$ It is expected that the indication of ranibizumab will be extended to CNV secondary to all causes other than age with time, as occurred with laser photocoagulation and PDT.

\section{Bevacizumab (Avastin)}

Bevacizumab (Avastin) is a full recombinant humanised monoclonal antibody with a molecular weight of $149 \mathrm{kD}$ (three times the size of ranibizumab [49kD]) that binds to all isoforms of VEGF-A (similar to ranibizumab). ${ }^{86}$ It is glycosylated, unlike ranibizumab, and has an Fc fragment, again unlike ranibizumab. The Fab domain of bevacizumab differs from that of ranibizumab by six amino acids. ${ }^{87,88}$ The serum and vitreous half-lives of bevacizumab are longer than those of ranibizumab..$^{89,90}$ The biological similarity of bevacizumab to ranibizumab has led to widespread 'off-label' use of bevacizumab for the treatment of wet AMD. These case series suggest that bevacizumab may be successful in the short term in terms of limiting visual loss. This is because of the perceived low cost of compounded bevacizumab compared with ranibizumab and apparently similar efficacy in the short term. A systematic review of bevacizumab in wet AMD has been undertaken by Schouten et al. ${ }^{91}$ however, as yet there are no reported large randomised clinical trials available for intraocular use of bevacizumab. As such, there are no safety data, and the minimum effective dose, optimum dose and dose frequency are not known. Unfortunately, the safety data of bevacizumab cannot be completely inferred from those of ranibizumab. The recent reports of severe intraocular inflammation associated with bevacizumab illustrate the point. ${ }^{92}$

\section{Comparison of Bevacizumab and Ranibizumab}

A number of trials around the world are directly comparing both molecules. The Comparison of Age-related Macular Degeneration Treatments Trial (CATT) in the US, Inhibit VEGF in Age-related Choroidal Neovascularisation (IVAN) in the UK, the Groupe d'Evaluation Francais d'Avastin vs Lucentis in France (GEFAL) studies and other similar studies are not due to report until 2011 or later. As non-inferiority trials with pre-defined margins of five ETDRS letter difference, these trials may reveal interesting data with respect to efficacy. However, all of these trials have significant trial design faults as they are underpowered to determine safety issues.

Both bevacizumab and ranibizumab block all VEGF isoforms. ${ }^{87}$ However, bevacizumab is a full-length immunoglobulin while ranibizumab is the antigen-binding fragment (Fab) portion of the molecule containing the specific active binding site of the antibody. The preparations of the two drugs have different $\mathrm{pHs}$ : ranibizumab has a pH of 5.5 while that of bevacizumab is 6.2. ${ }^{78,93}$ Ranibizumab penetrates the retina and inner limiting membrane more readily than bevacizumab and has a lower systemic half-life than the larger bevacizumab: the systemic half-life of bevacizumab is 20 days compared with two hours for ranibizumab. ${ }^{78,93}$ The longer half-life of bevacizumab may have significant implications for the development of ATEs compared with ranibizumab. Systemic bevacizumab has also been associated with increased blood pressure in some studies, ${ }^{93}$ while pooled data from ranibizumab studies revealed no significant change in blood pressure. ${ }^{73,77}$ There are reports of severe intraocular inflammation associated with bevacizumab in Canada and other parts of the world. ${ }^{92}$ The SAILOR study showed no statistical difference in ATEs between the different ranibizumab doses.$^{73}$ Cutaneous wound healing may also be affected differently. ${ }^{94}$

Ranibizumab also has a 100-fold greater affinity for VEGF than bevacizumab (binding affinity [I $\left.\mathrm{C}_{50}\right]$ to VEGF is $0.15 \mathrm{nM}$ for ranibizumab compared with $1.1 \mathrm{nM}$ for bevacizumab), with resultant greater potency in bioassays. ${ }^{78,93}$ Bevacizumab is thought to accumulate in the RPE cells, and is associated with Fc-mediated RPE cell death..$^{95}$ The theoretical risks of RPE retention and longer serum half life of bevacizumab compared with ranibizumab ${ }^{87,95,96}$ may imply potentially higher ocular and systemic adverse events, including RPE atrophy, subfoveal fibrosis and RPE tears. Recent studies have reported that there are significant differences in the immunoglobulin $G(I g G)$ content in pre-packaged bevacizumab syringes, and that micronsized protein aggregates increase with the attendant decrease in IgG content of the syringes. Such large aggregates may lead to aqueous outflow obstruction with subsequent intraocular pressure rise. ${ }^{97}$

In effect, there are no safety data available for intravitreal bevacizumab. Furthermore, there are no formal avenues for reporting adverse events associated with bevacizumab in most countries. Reporting mechanisms for adverse events are unclear, and even where such avenues exist, reports are arbitrary and subject to physician discretion. It is therefore highly possible that there is underreporting of adverse events.

\section{Combination Therapy with Photodynamic Therapy and Anti-vascular Endothelial Growth Factors}

It was postulated that combination therapy with PDT and anti-VEGFs may prove to be even more effective than either therapy on its own as the two treatments had different, probably complementary, mechanisms of action. Potentially, such combinations will improve efficacy, reduce frequency of re-treatments and reduce toxicity. However, the combination of pegaptanib and PDT (Macugen, Pfizer/OSI Eyetech EOP1012) has been disappointing as no difference was observed between the monotherapy and the therapy groups, although there were no safety concerns (Pfizer, data on file).

Results from the FOCUS study ${ }^{98}$ and the PROTECT study ${ }^{99}$ showed that the combination of PDT and ranibizumab was safe and that re-treatment rates may be reduced, with good outcomes. ${ }^{98,99}$ Similar safety studies had been previously reported in monkeys. ${ }^{100}$ Randomised controlled trials of such combinations, including the SUMMIT (MONT BLANC, DENALI, EVEREST) combinations of PDT and ranibizumab, have been completed and are awaiting publication. The preliminary data 
from these phase III trials of combination therapy of ranibizumab and PDT in wet AMD have not lived up to the promise of significantly reducing the frequency of injections as well as improving visual outcomes, except in EVEREST (for IPCV), where there was a significant difference in treatment frequency and outcome with combination therapy compared with monotherapy with either ranibizumab or PDT. ${ }^{101-103}$ No safety concerns were noted with the combination therapy.

However, it is implied that patients who have failed to respond to PDT may be safely transferred to anti-VEGF therapy without any risk. Similarly, patients unresponsive to ranibizumab monotherapy may benefit from combination therapy with PDT.

\section{Future Developments and Applications}

Despite its cost, ranibizumab, especially when given every month, is considered the gold standard in the treatment of CNV secondary to wet AMD. Combination therapy of ranibizumab and PDT is under investigation at present. However, monthly dosing of ranibizumab and other anti-VEGF agents as treatment of AMD presents a significant logistical challenge to clinical services worldwide. Significant strides would be made with treatment regimes that result in reductions in patient traffic through AMD clinics.

Extended-release preparations that will deliver ranibizumab over prolonged periods (three to six months) are desirable, as such systems will remove the need for repeated monthly intravitreal injections. Such development will improve the logistical bottlenecks in service delivery, as well as reducing injection-related risks such as endophthalmitis, retinal detachment and cataracts. However, the enthusiasm for such systems has to be tampered with the potentia increased risk of the undesirable effects of chronic VEGF antagonism in the neuro-retina, as VEGF is known to be neurotrophic..$^{104}$

There is hardly any competition from pegaptanib at the moment (at least in the UK), as it is considered less efficacious than ranibizumab in the treatment of wet AMD, and therefore is not recommended by NICE. However, in the presence of very high cardiovascular risk or known/ suspected allergy to ranibizumab, it would seem prudent to advise pegaptanib, even in the absence of direct evidence of comparisons.

Aflibercept (VEGF Trap, Regeneron Pharma/Bayer Schering) is a new molecule designed to block VEGF-mediated angiogenesis. It contains immunoglobulin domains of both VEGFR-1 and VEGFR-2 fused to a constant region, ${ }^{105}$ and neutralises VEGF and PIGF. This dual action may be an advantage over simple VEGF blockade. Phase I and II trials indicated no safety concerns, and there were VA gains and OCT reduction in central retinal thickness. ${ }^{106,107}$ It is possible that the treatment intervals may be slightly less frequent than with ranibizumab.

Pre-clinical and phase I studies have indicated that PDGF-B blockage may give increased efficacy in abrogating CNV, especially in combination with anti-VEGFs. ${ }^{108}$ The role of radiotherapy in combination with anti-VEGF therapy is yet to be fully assessed.

\section{Visual Rehabilitation}

For patients with advanced dry or wet AMD unresponsive to treatment, visual rehabilitation offers the potential of maximising the use of the patient's remaining vision. Low-vision clinics are available in most hospital optometry units and some community optometrist services. These services encompass advice on large-print books, optimum lighting and the use and provision of hand- and spectaclesupported magnifying devices and closed-circuit television (CCTV) devices. More recently, a number of training courses have become available for patients to learn to use eccentric fixation outside the area affected by disease for tasks such as reading.

\section{Referral Pathways}

The advent of new anti-VEGF therapies for wet AMD has led to success in stabilising vision in the vast majority of patients and the possibility of restoring vision lost due to the disease process in some patients. It has therefore become extremely important that patients reporting new symptoms, such as distortion or blurring in their central vision, are rapidly referred to a local ophthalmology department with facilities and experience to evaluate and treat AMD. This rapid referral and treatment are important as it is known that the treatment outcome is dependent on VA at treatment. It is recommended that AMD treatment providers institute referral pathways for general practitioners and optometrists in their locality. Options may include setting up rapid referral clinics with direct access for optometric or general ophthalmologist referrals, an open-access eye casualty/ emergency service or general practitioners with a special interest who can screen symptomatic patients.

\section{Age-related Macular Degeneration Service Specifications}

A diagnosis of CNV should be confirmed by FFA, except in cases of allergy that preclude such investigation, and OCT (Stratus OCT 3 equivalent or higher specification) before commencement of therapy. This is important in excluding potential simulating conditions and in re-defining the disease should there be non-response. ICG-A is recommended in as many cases as possible in order to determine the presence of idiopathic polypoidal choroidopathy, as these may not respond as well as CNV to anti-VEGF monotherapy compared with combined therapy (with anti-VEGF and PDT, or PDT monotherapy). Similarly, LogMAR VA measurements are recommended for AMD services rather than Snellen with or without conversion. A vision rehabilitation service should be closely linked to the AMD diagnosis and treatment service.

\section{Recommendations for Clinical Practice}

Patients with dry and wet AMD should be advised to stop smoking. Dry AMD patients should be advised to eat balanced diets, which may be enhanced with ocular nutritional supplements. Antismoking advice must be given to patients with all types of AMD. A healthy lifestyle must be stressed. Supportive measures such as low-vision aids cannot be overemphasised. The provision of, and advice on the use of, optical aids, and also counselling, are helpful in both dry and wet $A M D$.

It is recommended that ophthalmologists exercise caution and use their own judgement and experience, which must be underpinned by the existing evidence base when recommending treatments for wet AMD. The guiding principle should be that whatever treatment is recommended must be in the best interest of the patient. This clinical judgement should not be over-ridden by the perceived low cost of compounded bevacizumab compared with ranibizumab, pegaptanib or any other licensed product.

Intravitreal anti-VEGF may be used to treat all lesion types: classic, predominantly classic, minimally classic, occult and RAP lesions. 
Where anti-VEGF monotherapy fails, a switch of agent is sometimes useful. A combination of PDT and anti-VEGF should be considered in cases of non-response ('anti-VEGF resistance') as salvage, after reviewing the diagnosis.

Although the NICE guidance (in the UK) recommends treatment in eyes with VA between LogMAR 1.2 and 0.3 (6/96 and 6/12), these limitations are based on the pivotal clinical trials available at the evaluation of the technology. Post-marketing experience indicates that all lesion types with all levels of VA benefit from treatment with ranibizumab. It is known that eyes with better pre-treatment VA have better VA outcomes compared with those with worse baseline VA. Furthermore, it is considered inappropriate to allow the VA to deteriorate from better levels to 6/12 or worse in anyone before recommending treatment, as visual loss in these eyes is not linear and there is no guarantee of returning the VA to levels existing before the drop in vision occurred.

It is also accepted that most patients with wet AMD have a degree of foveal damage. As such, foveal damage cannot be a factor excluding treatment with anti-VEGFs. The degree of foveal damage that may prevent any benefits to treatment needs to be assessed by the treating clinician. This is particularly important as we know that treatment with anti-VEGFs prevents the further visual loss that would occur without any treatment. Prevention of further visual loss is accepted as a benefit of treatment.

\section{Lesion Type and Location}

\section{Extrafoveal Choroidal New Vessels}

Patients with small extrafoveal classic CNV should be treated with focal laser photocoagulation as described in the MPSG protocol. However, in patients with large extrafoveal classic CNV or occult/ minimally classic CNV with progression, it is justifiable to offer alternative treatment similar to that for juxtafoveal lesions. Where no progression is demonstrable or vision is not threatened, judicious observation may be advised. Laser photocoagulation is not recommended for patients with subfoveal or juxtafoveal CNV.

\section{Subfoveal/Juxtafoveal Choroidal New Vessels}

All eyes with subfoveal/juxtafoveal CNV of all lesion types will benefit from treatment.

Predominantly Classic Subfoveal/Juxtafoveal Choroidal New Vessels Anti-VEGF treatment is the recommended first-line treatment. However, patients with predominantly classic AMD with subfoveal and juxtafoveal location could be offered PDT or combination treatment in the first instance if regular attendance at a clinic is difficult. Where there is poor response to PDT in the treated eye, or in the other eye previously, treatment with licensed anti-VEGFs is recommended where available. In the absence of such availability, the use of unlicensed products, including bevacizumab, may be justified.

\section{Occult Subfoveal/Juxtafoveal Choroidal New Vessels}

The use of anti-VEGF is recommended. There are no other treatments of proven efficacy for these lesions.

Minimally Classic Subfoveal/Juxtafoveal Choroidal New Vessels Intraocular injections of anti-VEGF agents are considered first-line treatment for these lesions. PDT is not recommended for such patients.

\section{Retinal Angiomatous Proliferations}

RAPs occur commonly, and do not respond well to PDT monotherapy. It is recommended that eyes with RAPS as part of the CNV lesion are treated with repeated injections of anti-VEGFs, or combination therapy of anti-VEGF and PDT.

\section{Idiopathic Polypoidal Choroidopathy}

IPVC may occur alongside other CNV lesion types. High suspicion is therefore required for their detection. Normally, only patients with macular involvement are treated, unless central vision is threatened by persistent or progressive exudation. The threat of pre-retinal or vitreous haemorrhage should encourage treatment rather than observation. Direct laser photocoagulation can be used for extrafoveal IPCV. Active and symptomatic IPCV with subfoveal lesions can be treated with PDT or anti-VEGFs. However, IPCV lesions are more likely to respond to combination therapy with PDT and ranibizumab than to monotherapy with either agent. It is therefore recommended that whenever possible such combinations are given rather than monotherapy. When recommending intraocular bevacizumab (Avastin), it is extremely important to inform patients that it is unlicensed for this indication and that it has not undergone the usual rigorous clinical trials and independent evaluation by regulatory authorities. Adequate follow-up information should be collected as part of an ongoing prospective audit.

\section{Summary}

Treatments for wet AMD have improved considerably over the past decade. These improvements are expected to considerably reduce visual loss over time. However, there are significant resource implications. Attention is now being focused on dry AMD while refinements to wet AMD therapy continue. Visual rehabilitation remains a vital part of the management of $A M D$

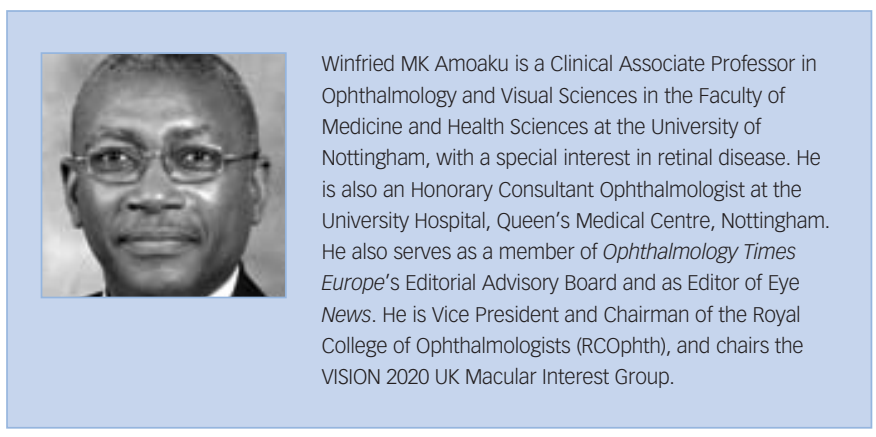

1. Ferris FL III, Fine SL, Hyman L, Age-related macular degeneration and blindness due to neovascular maculopathy, Arch Ophthalmol, 1984;102:1640-42.

2. Klein R, Klein B, Linton RL, Prevalence of age-related maculopathy. The Beaver Dam Eye Study, Ophthalmology, 1992;99:933-43.

3. Evans J, Causes of blindness and partial sight in England and Wales: 1990-1991, London: Her Majesty's Stationery Office, 1995.

4. Mitchell P, Smith W, Attebo K, Wang JJ, Prevalence of age-related maculopathy in Australia. The Blue
Mountains Eye Study, Ophthalmlology, 1995:102:1450-60

5. Bonastre J, Le PC, Anderson P, et al., The epidemiology, economics, and quality of life burden of age related macular degeneration in France, Germany, Italy and the United Kingdom, Euro J Health Econ, 2002;3:94-102.

6. Sarks $\mathrm{SH}$, Ageing and degeneration in the macular region, Br J Ophthalmol, 1976;60:324-41.

7. Green WR, Enger $C$, Age-related macular degeneration histopathologic studies. The 1992 Lorenz E Zimmerman Lecture, Ophthalmlogy, 1993;100:1519-35.

8. Avery RJ, Fekrat S, Hawkins BS, Bressler NM, Natural history of subfoveal subretinal haemorrhage in age related macular degeneration, Retina, 1996;16:183-9. 9. Grossniklaus HE, Green WR, Choroidal neovascularisation, Am J Ophthalmol, 2004;137:496-503.

10. Olsen TW, Feng $X$, Kasper TJ, et al., Fluorescein angiographic lesion type frequency in neovascular agerelated macular degeneration, Ophthalmology, 2004;111: 250-55.

11. Kvanta A, Algvere PV, Berglin L, Seregard S, Subfoveal fibrovascular membranes in age-related macular degeneration express vascular endothelial growth factor, 
Invest Ophthalmol Vis Sci, 1996;37:1929-34.

12. Lopez PF, Sippy BD, Lambert HM, et al , Trandifferentiated retinal pigment epithelial cells are immunoreactive for vascular endothelial growth factor in surgically excised age-related macular degenerationrelated choroidal neovasacular membranes, Invest Ophthalmol Vis Sci, 1996;37:855-68.

13. Frank RN, Amin RH, Eliot $\mathrm{D}$, et al., Basic fibroblast growth factor and vascular endothelial growth factor are present in epiretinal and choroidal neovascular membranes, Am J Ophthalmol, 1996;122:393-403

14. Cui JZ, Kimura H, Spee C, et al., Natural history of choroidal neovascularisation induced by vascula endothelial growth factor in the primate, Graefe Arch Clin Exp Ophthalmol, 2000;238:326-33.

15. Bezerra Y, Fuselier JA, Peyman GA, et al., Study of inhibitory effects of an antiangiogenic somatostatincamptothecin conjugate on laser induced choroida neovascularisation in rats, Retina, 2005;25:345-54.

16. Jo N, Mailhos C, Ju M, et al., Inhibition of platelet-derived growth factor $\mathrm{B}$ signalling enhances the efficacy of antivascular endothelial growth factor therapy in multiple models of ocular neovascularisation, Am J Pathol, 2006;168:2036-53.

17. Rackic JM, Lambert V, Devy L, et al., Placental growth Factor, a member of the VEGF family, contributes to the development of choroidal neovascularisation, Invest Ophthalmol Vis Sci, 2003;44;3186-93.

18. Owen $C G$, Fletcher AE, Donoghue M, Rudnicka AR, How big is the burden of visual loss caused by age related macular degeneration in the United Kingdom?, Br J Ophthalmol, 2003:87(3):312-17.

19. NICE, HTA Ranibizumab and Pegaptanib for the treatment of AMD. Final Scope. Available at: www.nice.org.uk (accessed 25 April 2010).

20. Macular Photocoagulation Study Group, Risk factors for choroidal neovascularisation in the second eye of patients with juxtafoveal or subfoveal choroidal neovascularisation secondary to age-related macular degeneration, Arch Ophthalmol 1997:115:741-7.

21. Bird AC, Bressler NM, Bressler SB, et al., The international ARM epidemiological study group. An international classification and grading system for agerelated maculopathy and age-related macular degeneration, Surv Ophthalmol, 1995;39:367-74.

22. Khan JC, Thurlby DA, Shahid H, et al., Smoking and agerelated macular degeneration: the number of pack years of cigarette smoking is a major determinant of risk for both geographic atrophy and choroida neovascularisation, Br J Ophthalmol, 2006;90:75-80.

23. Klein R, Knudtson MD, Cruickshanks KJ, Klein BE, Further observations on the association between smoking and long-term incidence and progression of age-related macular degeneration: The Beaver Dam Study, Arch ophthalmol, 2008;126:115-21.

24. Bressler SB, Maguire MG, Bressler NM, Fine SL, Macula Photocoagulation Group. Relationship of drusen and abnormalities of the retinal pigment epithelium to the prognosis of neovascular macular degeneration, Arch Ophthalmol, 1990;108:1442-7.

25. Mullins RF, Russell SR, Anderson DH, Hageman GS, Drusen associated with aging and age-related macular degeneration contain proteins common to extracellular deposits associated with atheroscerosis, elastosis, amyloidosis, and dense deposit disease, FASEB J, 2000;14:835-46.

26. Hageman GS, Anderson $\mathrm{DH}$, Johnson LV, et al., A common haplotype in the complement regulatory gene factor $\mathrm{H}$ (HF1.CFH) predisposes individuals to age-related macula degeneration, Proc Natl Acad Sci, 2005;102:7227-32.

27. Klein RJ, Zeiss $\mathrm{C}$, Chew EY, et al., Complement factor $\mathrm{H}$ polymorphism in age-related macular degeneration, Science, 2005;308:385-9.

28. Edwards AO, Ritter R 3rd, Abel KJ, et al., Complement factor $\mathrm{H}$ polymorphism and age-related macular degeneration, Science, 2005;308:421-4.

29. Haines JL, Hauser MA, Schmidt S, et al., Complement factor $\mathrm{H}$ variant increases the risk of age-related macula degeneration, Science, 2005;308:419-21

30. Rivera A, Fisher SH, Fritsche LG, et al., Hypothetical Loc
387715 is a second major histocompatibility gene for age-related macular degeneration, contributing independently of complement factor $\mathrm{H}$ to disease risk, Human Mol Genet, 2005:14:3227-36.

31. DeWan A, Liu, M, Hartman $\mathrm{S}$, et al., HTRA1 polymorphism in wet age-related macular degeneration science, 2006;314:989-92.

32. Yang Z, Camp NJ, Sun $\mathrm{H}$, et al., A variant of the HTRA gene increases susceptibility to age-related macular degeneration, Science, 2006:314:992-3.

33. Gold B, Merriam JE, Zernant J, et al., Variations in factor $\mathrm{B}(\mathrm{BF})$ and complement component 2 (C2) genes is associated with age-related macular degeneration, Nat Genet, 2006;38:458-62.

34. Commissioning Contemporary AMD Services RCOphth, 2007. Available at: www.rcophth.ac.uk/docs/ publications/published-guidelines/CommissionContemp AMDServicesV3Final.pdf (accessed 14 May 2010).

35. RCophth AMD Guidelines, 2009. Available at: www.rcophth.ac.uk/docs/publications/AMD_GUIDELINES FINAL VERSION Feb 09.pdf (accessed 14 May 2010).

36. Yannuzzi LA, Negrao $S$, lida $T$, et al., Retinal angiomatous proliferation in age related macular degeneration, Retina, 2001:21:416

37. Monson, DM, Smith JR, Klein ML, Wilson DJ, Clinicopathologic correlation of retinal angiomatous proliferation, Arch Ophthalmol, 2008;126(12):1664-8.

38. Scott AW, Bressler SB, Retinal angiomatous proliferation or retinal anastomosis to the lesion, Eye (Lond), 2010;24(3): 491-6.

39. Massacesi AL, Sacchi L, Bergamini F, Bottoni F, The prevalence of retinal angiomatous proliferation with occult choroidal neovascularisation, Graefe Arch Clin Exp ophthalmol, 2008:246:89-92.

40. Gupta BM, JyothiS, Sivaprasad S, Current treatmen options for retinal angiomatous proliferans (RAP) Br I Ophthalmol, 2009 (Epub ahead of print).

41. Yannuzzi LA, Sorenson J, Spaide RF, Idiopathic polypoidal choroidal vasculopathy, Retina, 1990;10:1-8.

42. Age-Related Eye Disease Study Research Group (AREDS), Risk factors associated with age-related macular degeneration. A case-control study in the age-related eye disease study: Age-related Eye Disease Study Report Number 3, Ophthalmology, 2000;107:2224-32.

43. Age-Related Eye Disease Study Research Group, A randomised, placebo-controlled, clinical trial of high dose supplementation with vitamins $\mathrm{C}$ and $\mathrm{E}$, beta carotene, and zinc for age-related macular degeneration and vision loss : AREDS report no. 8, Arch Ophthalmol, 2001;119:1417-36.

44. AREDS 2 Trial. Available at: www.nei.nih.gov/neitrials/ viewstudyWeb.aspx?id=120 (accessed 15 February 2007).

45. Macular Photocoagulation Study Group, Argon laser photocoagulation for neovascular maculopthy. Three year results from randomised clinical trials, Arch Ophthalmol 1986:104:694-701.

46. Macular Photocoagulation Study Group, Recurrent choroidal neovascularisation after argon laser photocoagulation for neovascular maculopathy Arch Ophthalmol, 1986;104:503-12.

47. Macular Photocoagulation Study Group, Lase photocoagulation of subfoveal neovascular lesions in age-related macular degeneration, Arch Ophthalmol, 1991;109:1220-31.

48. Macular Photocoagulation Study Group, Visual outcome after laser photocoagulation for subfoveal choroida neovascularisation secondary to age-related macula degeneration. The influence of initial lesion size and initial visual acuity, Arch Ophthalmol, 1994;112:480-88.

49. Treatment of Age-Related Macular Degeneration with Photodynamic Therapy (TAP) Study Group, Photodynamic therapy of subfoveal choroidal neovascularisation in age related macular degeneration with verteporfin: one year results of 2 randomised trials - TAP report 1, Arch Ophthalmol 1999:117:1329-45.

50. Treatment of Age-Related Macular Degeneration with Photodynamic Therapy (TAP) Study Group, Photodynamic therapy of subfoveal choroidal neovascularisation in age related macular degeneration with verteporfin: two-year results of 2 randomised clinical trials - TAP report 2, Arch
Ophthalmol, 2001;119:198-207

51. Verteporfin in Photodynamic Therapy Study Group Verteporfin therapy of subfoveal choroida neovascularisation in age-related macular degeneration: two-year results of a randomised clinical trial including lesions with occult with no classic choroida neovascularisation - Verteporfin in Photodynamic Therapy report 2, Am J Ophthalmol, 2001;131:541-60.

52. Verteporfin in Photodynamic Therapy Study Group, Photodynamic therapy of subfoveal choroidal neovascularisation in pathologic myopia with verteporfin one-year results of a randomised clinical trial including lesions with occult with no classic choroidal neovascularisation - VIP Report 1, ophthalmology, 2001;108:841-52

53. Ishida S, Isui T, Yamashiro K, et al., VEGF164-mediated inflammation is required for pathological but not physiological, ischemia-induced retin neovascularisation, J Exp Med, 2003;198:483-9.

54. Gragoudas ES, Adamis AP, Cunningham ET Jr, et al.; VEGF Inhibition Study in Ocular Neovascularisation Clinical Trial Group, N Eng/ J Med, 2004;351:2805-16.

55. VISION Clinical Trial Group (D'Amico DJ et al), Pegaptanib sodium for neovascular age-related macular degeneration: two year safety results of the two year prospective, multicentre, controlled clinical trials, ophthalmology, 2006;113:992-1001.

56. Feucht N, Mattias H, Lohmann CP, Maier M, Pegaptanib sodium treatment in neovascular age-related macular degeneration: clinical experience in Germany, Clin Ophthalmol, 2008:2:253-9.

57. Rosina C, Bottoni F, Staurenghi G, Cllinical experience with pegaptanib sodium, Clin Ophthalmol, 2008;2:485-8.

58. Sivaprasad S, Acharya N, Pegaptanib sodium for neovascular age-related macular degeneration: clinical experience in the UK, Clin Ophthalmol, 2008;2:347-54.

59. Steffensmeier AC, Fuller JJ, Muller BA, Russell SR, Severe systemic allergic responses associated with vitreous injections of Pegaptanib sodium, Invest Ophthalmol Vis Sci. 2006; 47: E-abstract 4247.

60. Chang LK, Shah S, Chen J, Sarraf D, OCT imaging of RPE tears in patients receiving treatment with Macugen for AMD-related PED, Invest Ophthalmol Vis Sci, 2006;47: E-abstract 2185.

61. Rosenfeld PJ, Brown DM, Heier JS, et al.; for the MARINA Study Group, Ranibizumab for Neovascular Age-Related Macular Degeneration, N Eng/ J Med, 2006:355:1419-31.

62. Brown DM, Kaiser PK, Michels $\mathrm{M}$, et al.; for the ANCHOR Study Group Subgroup Analysis, Ranibizumab vrs Verteporfin for Neovascular Age-Related Macular Degeneration, N Engl J Med, 2006;355:1432-44.

63. Regillo CD, Brown DM, Abraham P, et al.; for the PIER Study Group, Randomized, Double-Masked, ShamControlled Trial of ranibizumab for neovascular agerelated macular degeneration: PIER Study Year 1, Am J ophthalmol, 2008:145:239-48.

64. Brown DM, Michels M, Kaiser PK, et al.; for the ANCHOR Study Group, Ranibizumab versus verteporfin photodynamic therapy for neovascular age-related macular degeneration. Two year results of the ANCHOR Study, Ophthalmology, 2009;116:57-65.

65. Miller JW, Shapiro H, Acharya N; for the MARINA Study Group, Randomized, Controlled Phase III Study of Ranibizumab (Lucentis) for Minimally Classic or Occult Neovascular Age-Related Macular Degeneration: TwoiYear Safety Results of the MARINA Study, Invest Ophthalmol Vis Sci, 2006;47: E-abstract 3539.

66. Boyer DS, Antoszyk AN, Awh CC, et al., Subgroup analysis of the MARINA Study of ranibizumab in neovascular age-related macular degeneration, Ophthalmology, 2007;114:246-52.

67. Kaiser PK, Brown DM, Zhang K, et al., Ranibizumab for predominantly classic neovascular age-related macular degeneration: subgroup analysis of first-year ANCHOR results, Am I Ophthalmol, 2007:144:850-57.

68. Kaiser PK, Blodi BA,Shapiro H, Acharya NR; MARINA Study Group, Angiographic and optical coherent tomographic results of the MARINA Study of ranibizumab in neovascular AMD, Ophthalmology, 2007;114:1868-75.

69. Chang TS, Bressler NM, Fine JT, et al.; for the MARINA 
Study Group, Improved vision-related function afte ranibizumab treatment for neovascular age-related macular degeneration, Arch Ophthalmol, 2007;125: 1460-69.

70. Bressler NM, Chang TS, Fine JT, et al.; for the ANCHOR Research Group, Improved vision-related function after ranibizumab versus photodynamic therapy: a randomised clinical trial, Arch Ophthalmol, 2009;127:13-21.

71. Meyer CH, Eter N, Holz FG; the SUSTAIN Study Group, Ranibizumab in patients with subfoveal choroida neovascularisation secondary to age-related macular degeneration. Interim results from the SUSTAIN Trial, Invest Ophthalmol Vis Sci, 2008;49: E-abstract 273.

72. Ferrone PJ, Francom S, Toumi L; for the SAILOR Group, Efficacy of ranibizumab (Lucentis) treatment in patients with neovascular AMD: subgroup analysis of SAILOR Study Cohort 1, Proceedings of ASRS 2008. Available at: www.medscape.com/viewarticle/583347 (accessed 30 March 2010).

73. Boyer DS, Heier JS, Brown DM, et al., A Phase IIIb study to evaluate the safety of ranibizumab in subjects with neovascular age-related macular degeneration, Ophthalmology, 2009;116:1731-9.

74. Collaborative overview of randomized trials of antiplatelet therapy - I: Prevention of death, myocardial infarction, and stroke by prolonged antiplatelet therapy in various categories of patients. Antiplatelet Trialists Collaboration, BMJ, 1994;308:81-106.

75. Genentech Inc., Dear Healthcare provider letter regarding Lucentis. Available at: www.fda.gov/ medwatch/safety/2007/Lucentis_DHCP_01-24-2007.pdf (accessed 2 May 2010)

76. US Food and Drug Administration, 2007 Safety alerts for drugs, biologics, medical devices, and dietary supplements. Available at: www.fda.gov/medwatch/ safety/2007/safety07.htm (accessed 25 April 2010).

77. Novartis, data on file. Meta-analysis of MARINA, ANCHOR, PIER, SAILOR Safety analysis, 2009.

78. European Medicines Agency, Ranibizumab (Lucentis): Summary of product characteristics. Available at: www.emea.europa.eu/humandocs/PDFs/EPAR/lucentis/ H-715-PI-en.pdf (accessed 25 April 2010).

79. Amoaku W, Ranibizumab: The clinician's guide to commencing, continuing and discontinuing treatment, Eye, 2009;23:2140-42

80. National Institute for Health and Clinical Excellence (NICE) TA 155, Evaluation of ranibizumab and pegaptanib for the treatment of wet age-related macular degeneration, 2008. Available at: www.nice. org.uk/Guidance/TA155/Guidance/pdf/English (accessed 25 April 2010)

81. Atmani $\mathrm{K}$, Voigt $\mathrm{M}$, Le Tien $\mathrm{V}$, et al., Ranibizumab for retinal angiomatous proliferation in age-related macular degeneration, Eye, 2010;24(7):1193-9.

82. Lai T, Chan WM, Liu DT, Lam DS, Ranibizumab for retin angiomatous proliferation in neovascular age-related macular degeneration, Graefe Arch Clin Exp Ophthalmol, 2007;245:1877-80

83. Silva RM, Ruiz-Moreno JM, Nascimento J, et al., Shortterm efficacy and safety of intravitreal ranibizumab for myopic choroidal neovascularisation, Retina, 2008; 28:1117-23.

84. Konstatinidis L, Mantel I, Pounaras JA, et al., Intravitreal ranibizumab (Lucentis) for the treatment of myopic choroidal neovascularisation, Graefe Arch Clin Ophthalmol, 2009;247:1567-9.

85. Cohen SY, Anti-VEGF drugs as the 2009 first-line therapy for choroidal neovascularisation, Retina, 2009;29:1062-6.

86. Presta LG, Chen H, O'Connor SJ, et al., Humanisation of an anti-vascular endothelial growth factor monoclonal antibody for the therapy of solid tumors and other disorders, Cancer Res, 1997:57:4593-9.

87. Ferrara N, Damico L, Shams N, et al., Development of ranibizumab, an anti-VEGF antigen binding fragment, as therapy for neovascular macular degeneration, Retina, 2006:26:859-70

88. Kim KJ, Li B, Winer J, et al., Inhibition of vascular endothelial growth factor-induced angiogenesis suppresses tumor growth in vivo, Nature, 1993;362:841-4.

89. Bakri SJ, Snyder MR, Reid JM, et al., Pharmacokinetics of intravitreal bevacizumab (Avastin), Ophthalmology, 2007: 114:855-9.

90. Bakri SJ, Snyder MR, Reid JM, et al., Pharmacokinetics of intravitreal ranibizumab (Lucentis), Ophthalmology, 2007;114:2179-82

91. Schouten JS, La Heij EC, Webers CA, et al., A systematic review on the effect of bevacizumab in exudative agerelated macular degeneration, Graefes Arch Clin Exp Ophthalmol, 2009:247:1-11.

92. Georgopoulos M, Polak K, Pruente C, Schmidt-Erfurth U, Characteristics of severe inflammation of following intravitreal injection of bevacizumab, $\mathrm{Br} J$ Ophthalmol, 2009;93:457-62

93. European Medicines Agency, Bevacizumab (Avastin): Summary Product Characteristics. Available at: www.ema.europa.eu/humandocs/PDFs/EPAR/avastin/1 7199204en6.pdf (accessed 2 May 2010).

94. Christoforidis JB, Ricketts R, Bean S, et al., The effect of intravitreal pegaptanib, bevacizumab and ranibizumab on blood vessel formation during cutaneous wound healing in a rabbit model, Invest Ophthalmol Vis Sci, 2010; $51:$ E-Abstract 6150

95. Tezel TH, Zeng Q, Schaal S, Kaplan HJ, Bevacizumab (Avastin) differs from ranibizumab (Lucentis) in its binding affinity to retinal pigment epithelium (RPE) and vascular endothelium cell membranes, Invest ophthalmo
Vis Sci, 2010;51: E-Abstract 4955.

96. Zeng Q, Schaa SI, Kaplan HJ, Tezel TH, Ability to Bind FCReceptors on Retinal Pigment Epithelium (RPE) can explain the higher occurence of RPE tears with bevacizumab (Avastin) compared with ranibizumab (Lucentis), Invest Ophthalmol Vis Sci, 2010;51: E-Abstract 4954.

97. Ammar DA, Liu L, Ruzycki P, et al., High Molecular Weight Aggregates in Repackaged Bevacizumab, Invest Ophthalmol Vis Sci, 2010;51: E-Abstract 2816.

98. Heier JS, Boyer DS, Ciulla TA, et al.; FOCUS Study Group, Ranibizumab combined with verteporfin photodynamic therapy in neovascular age-related macular degeneration: year 1 results of the FOCUS Study, Arch Ophthalmol, 2006;124:1532-42.

99. Schmidt-Erfurth U, Wolf S; PROTECT Study Group, Same-day administration of verteporfin and ranibizumab $0.5 \mathrm{mg}$ in patients with neovascularisation due to age-related macular degeneration, Br J ophthalmol, 2008:92:1628-35

100. Husain D, Kim I, Gautlier D, et al., Safety and efficacy of intravitreal ranibizumab in combination with verteporfin photodynamic therapy on experimental choroida neovascularisation in the monkey, Arch Ophthalmol 2005;123:509-16.

101. Schmidt-Erfurth U, Summit Study Group. Proceedings of SOE, 2009. Novartis: data on file.

102. DENALI Study Group Study Design. Proceedings of SOE, 2009. Novartis, on file.

103. Lai TY; EVEREST Study Group, Verteporfin PDT and ranibizumab combination therapy for symptomatic macular polypoidal choroidal vasculopathy (PCV): EVEREST results, Invest Ophthalmol Vis Sci, 2010;51: ARVO E-abstract 2228.

104. D'Amore PA, Vascular endothelial cell growth factor-A Not just for endothelial cells anymore, Am I Pathol, 2007:171:14-18.

105. Holash J, Davis S, Papadopoulos N, et al., VEGF-Trap: VEGF blocker with potent antitumor effects, Proc Natl Acad Sci U S A, 2002:99:11393-8.

106. Nguyen QD, Shah SM, Browning DJ, et al., A Phase 1 study of endothelial growth factor trap-eye in patients with neovascular age-related macular degeneration, Ophthalmology, 2009;116:214-18.

107. Dixon JA, Oliver SC, Olson JL, Mandava N, VEGF Trap Eye for the treatment of neovascular age-related macular degeneration, Expert Opin Investig Drugs, 2009:18:1573-80

108. Jo N, Mailhos C, Ju M, et al., Inhibition of plateletderived growth factor $\mathrm{B}$ signalling enhances the efficacy of anti-vascular endothelial growth factor therapy in multiple models of ocular neovascularisation, Am J Pathol, 2006:168:2036-53. 